\title{
Échange polémique et contre-discours en italien
}

Quelques considérations sur les mécanismes langagiers et les stratégies discursives dans l'interaction conflictuelle

Polemical exchange and counter-discourse in Italian: reflections on linguistic mechanisms and discourse strategies in conflictual communication

\section{Alessandra Rollo}

\section{(2) OpenEdition}

Journals

Édition électronique

URL : http://journals.openedition.org/tipa/1950

DOI : 10.4000/tipa.1950

ISSN : 2264-7082

\section{Éditeur}

Laboratoire Parole et Langage

\section{Référence électronique}

Alessandra Rollo, «Échange polémique et contre-discours en italien », TIPA. Travaux interdisciplinaires sur la parole et le langage [En ligne], 33 | 2017, mis en ligne le 09 octobre 2017, consulté le 26 septembre 2020. URL : http://journals.openedition.org/tipa/1950 ; DOI : https://doi.org/10.4000/tipa. 1950

Ce document a été généré automatiquement le 26 septembre 2020.

\section{cc) (1) $९$}

La revue TIPA. Travaux interdisciplinaires sur la parole et le langage est mise à disposition selon les termes de la licence Creative Commons Attribution - Pas d'Utilisation Commerciale - Pas de Modification 4.0 International. 


\title{
Échange polémique et contre- discours en italien
}

\author{
Quelques considérations sur les mécanismes langagiers et les stratégies \\ discursives dans l'interaction conflictuelle \\ Polemical exchange and counter-discourse in Italian: reflections on linguistic \\ mechanisms and discourse strategies in conflictual communication
}

\section{Alessandra Rollo}

\section{Introduction}

1 D'après le spécialiste de la communication Dominique Wolton (2009), le défi du XXI siècle consiste à organiser la cohabitation pacifique de points de vue contradictoires où chacun veut conserver son identité; autrement dit, communication comme synonyme de cohabitation et de partage.

2 Or, dans le contexte actuel de conflictualité exacerbée, la société moderne devient fréquemment un théâtre de divergences et d'antagonismes - de nature politique, religieuse, idéologique, sociale - aussi bien entre individus et groupes au sein d'un même pays qu'entre peuples de nationalités différentes. Plusieurs échanges langagiers dans les espaces publics et privés se placent sous le signe du désaccord, de l'impolitesse, voire de la violence verbale, au point que le concept de «dialogue», quoique habituellement invoqué au nom d'une coopération harmonieuse régissant en principe tout échange verbal rationnel, semble être remis en cause au profit de celui de "débat». Un concept qui est très bien représenté par la métaphore structurale proposée par les cognitivistes Lakoff et Johnson (1980, tr. fr. 1985: 14 sqq.) : LA DISCUSSION, C'EST LA GUERRE, où le débat est structuré sous forme de lutte, les personnes avec qui l'on discute sont des adversaires, le débat/combat implique la mise en œuvre de stratégies grâce auxquelles on perd ou l'on gagne. Autant dire que l'activité discursive est structurée dans la culture occidentale en termes de bataille 
communicationnelle, d'affrontement de thèses, témoignant du fait que le conflit est, de gré ou de force, une composante incontournable de la réalité sociale. ${ }^{1}$

D'autre part, le discours conflictuel n'est pas qu'un cas particulier dans une typologie linguistique plus ou moins arbitraire, mais l'une des dimensions constitutives de toute société : une société se constitue autant dans et par le conflit, que par la collaboration et la coopération mutuelles. (Windish, $2007: 23$ )

Tout dialogue est à la fois coopératif et conflictuel, observe Kerbrat-Orecchioni (1986 : 24), le conflit n'étant pas un obstacle à la communication, mais la mise en place d'une différenciation des voix qui s'avère nécessaire à la poursuite de l'interaction (ibid.) et à l'affirmation de l'identité de chaque interactant.

Les discours politiques et médiatiques notamment, qui ont une forte connotation idéologique, présentent souvent un potentiel agressif plutôt marqué, étant animés par le désir d'influencer et de manipuler la pensée et les actions des récepteurs, bref, à agir sur les autres : le discours politique n'a pas de sens hors de l'action, et [...] dans l'action se joue, pour le sujet politique, l'exercice d'un pouvoir (Charaudeau, 2002). C'est dire que [l]e discours est une forme d'action (Maingueneau, 2010: 31), vu que parler n'est pas seulement représenter le monde, mais que c'est «faire quelque chose», c'est poser des actes de parole (ou actes de langage), comme l'ont postulé d'abord le philosophe du langage Austin dans son ouvrage fondateur Quand dire, c'est faire (1970) et puis son disciple Searle dans Les actes de langage. Essai de philosophie du langage (1972). Selon la théorie générale de la parole comme action, tous les énoncés sont investis d'une fonction pragmatique et servent à accomplir un certain acte social, y compris ceux qui véhiculent un contenu informationnel ou un sens cognitif et qui peuvent être qualifiés tout simplement de vrais ou de faux.

5 S'inscrivant dans cette perspective, notre contribution se propose de mettre en lumière quelques caractéristiques marquantes des mécanismes langagiers enclenchés lors d'une interaction conflictuelle. À cette fin, nous reprendrons les archétypes conceptuels d'agent-patient ${ }^{2}$, le discours conflictuel du sujet parlant se configurant comme un discours-agent (désormais DA) et le discours du sujet interpellé ou de l'adversaire étant un discours-patient (désormais DP) (Windisch, $1982: 106$; Maingueneau, $1983: 16$ ).

\section{Le conflit discursif : discours-agent vs discours- patient}

6 Au cours d'une conversation, on négocie sans cesse le sens de ce qu'on se dit ainsi que le type de rapport qu'on entretient tout au long de l'échange ; chaque participant tient à ce que l'image de lui qu'il y projette soit reconnue par l'autre. Breton (2000: 57) observe que, dans la société de communication, ne communiquent finalement que les images des entités qui le composent, des images conçue[s] presque exclusivement en termes de cause à promouvoir.

7 Cette identité interactionnelle spécifique que l'on s'accorde mutuellement et qui est dite face $^{3}$ (Brown \& Levinson, 1987) est centrale dans la communication verbale. Toute rencontre sociale est source de conflit potentiel, susceptible de rendre la face des sujets impliqués particulièrement vulnérable par la mise en doute de leurs qualités (face positive) ou la mise en danger de leur liberté (face négative). Lors de l'interaction, chacun essaie de protéger sa face, de ne pas la perdre, en comptant sur le respect de ses propres besoins et de son autonomie de la part des interlocuteurs. On aime être 
apprécié, et en même temps, on veut que les autres se sentent à l'aise, ce qui entraîne la mise en œuvre de contraintes rituelles et de stratégies conversationnelles, notamment de stratégies de politesse positives et négatives, visant à réduire les tensions qui risquent de surgir en présence de l'autre et à minimiser les actes menaçants pour la face des partenaires de l'échange langagier (FTAs ou Face Threatening Acts).

Le discours conflictuel témoigne du souci d'être reconnu et d'inspirer de la bienveillance chez les récepteurs, mais cela se fait au détriment de l'interlocuteur, en lui portant préjudice par l'impolitesse et l'agressivité. Si la politesse amène à se dominer, la violence vise à dominer autrui et à exercer une force pour contraindre l'autre à (ré)agir (Moïse \& Oprea, 2015). Au sein d'un échange agonique ou à dominante conflictuelle, où les règles de politesse sont violées et où l'on enfreint le principe coopératif préconisé par Grice (1975, tr. fr. 1979: 61 sqq.), la face de l'autre - son image publique, sa représentation sociale - est constamment menacée et devient la cible de critiques, d'insultes ou de paroles humiliantes.

Chaque acteur social engagé dans le débat peut occuper alternativement la position d'agent et de patient. Le sujet conceptuellement proéminent, qui parle et qui veut emporter l'adhésion de l'auditoire à ses propres thèses (agent), développe son argumentation par une réfutation du discours de l'adversaire (patient), au sens où il absorbe, se réapproprie, manipule ${ }^{4}$ et déforme la thèse opposée afin de la présenter sous le jour le plus défavorable, voire de la ridiculiser, ou bien retourne l'argument contre son adversaire ou lui oppose un contre-argument qui l'invalide (l'argumentation est donc évidemment polémique ${ }^{5}$ ). De son côté, le sujet interpellé, c'est-à-dire celui qui est impliqué dans l'altercation et qui subit à contre-gré l'attaque, est poussé à réagir, à rétorquer à son interlocuteur, en se transformant à son tour en agent et en adoptant les mêmes démarches discursives.

Cette dynamique déclenche une alternance entre discours-agent et discours-patient ${ }^{6}$, lesquels s'articulent dans un conflit discursif, visant à la légitimation du premier discours et à la délégitimation du second. Il s'agit d'un véritable duel verbal reposant sur un jeu d'assauts, d'autodéfense et de contre-attaques, dont la fonction est non seulement de combattre les thèses émises par l'autre, mais aussi de le décrédibiliser et de démolir son identité (sa face positive), afin de créer un consensus chez le public ${ }^{7}$.

On le devine, dans le cas de la communication conflictuelle, il ne s'agit plus seulement de transmettre sagement une information et de la faire comprendre le plus tranquillement du monde. Dire, informer et comprendre ne sont plus les objectifs essentiels [...]. D'autres objectifs peuvent devenir primordiaux, d'autres fonctions sociales de la langue peuvent compléter sa fonction première de communication [...]. La prise de parole peut servir à lutter, à combattre, à vaincre, à résister, à se révolter. (Windisch, $2007: 18$ )

11 Du fait qu'il reprend un autre discours pour s'y opposer, le discours conflictuel se profile comme un contre-discours, visant d'un côté à frapper la face de l'autre pour en proposer une représentation sociale péjorative et, de l'autre côté, à consolider sa propre image de crédibilité et de légitimité.

12 Le domaine politique est sans doute l'un des lieux d'élection de ce genre d'interaction. C'est que le discours se trouve être le plus manipulable des leviers d'action dont disposent les politiques (Bart, 1998: 52). Et c'est justement de la sphère institutionnelle-politique italienne que relèvent nos deux cas d'étude (un échange polémique et un contrediscours $\left.{ }^{8}\right)$, analysés dans ce qui suit. 


\section{Le cas d'un échange polémique} nous offre l'occasion d'illustrer les caractéristiques saillantes de la communication conflictuelle au niveau textuel et discursif, à partir du modèle élaboré par Windisch (1982; 2007). polémique représente l'une des variétés discursives (Dascal, 1998. Amossy, 2011), est bien une forme de combat - un combat verbal - incarnant parfaitement la métaphore de la guerre mentionnée plus haut; ce mot fonctionne comme une métaphore lexicalisée: une polémique, c'est une guerre métaphorique, une " guerre de plume» (Kerbrat-Orecchioni, 1980 : 4).

De même, la polémique s'inscrit dans la théorie des actes de langage :

Le genre polémique a donc ceci de spécifique, qui distingue son statut rhétorique de celui d'autres genres : la polémique est un acte, et non pas simplement un discours ; son dire est avant tout un faire ; un faire dont la portée - dans l'histoire littéraire comme dans l'histoire politique - ne coïncide pas nécessairement avec l'intention, la conscience du dire. (Felman, $1979: 182$ )

La visée pragmatique dominante d'un tel genre, comme le rappelle encore KerbratOrecchioni (1980:12), est de discréditer l'adversaire, et le discours qu'il est censé tenir.

Notre réflexion portera plus spécifiquement sur deux documents, datés du mois d'avril 2011, qui ont été envoyés aux citoyens de San Romano in Garfagnana, dans la province de Lucques: le premier document (DA) est celui de la minorité du conseil municipal (sujet-agent), qui adresse une série d'accusations à l'administration municipale à propos de la gestion et de la programmation financière; en réponse au premier document, fait suite un second (DP) du groupe de la majorité (sujet-patient, se transformant en sujet-agent), qui soutient le maire élu. Lesdits documents représentent donc un cas emblématique de cette forme d'activité dialogique conflictuelle qu'est l'échange polémique, où les adversaires (ou bien, les positions discursives qui s'affrontent) adressent des critiques l'un à l'autre à propos d'un sujet ou d'un problème sur lequel ils sont en désaccord (Dascal, 1998); comme les armes sont ici les arguments, on a affaire à des échanges d'arguments contre d'autres arguments (Oléron, 1995 : 17).

\subsection{Plan textuel : marques graphiques et lexicales}

18 Du point de vue de la forme textuelle, au nombre des marques linguistiques discrètes qui laissent entrevoir une controverse dialoguée, on peut détecter les marques graphiques du discours rapporté : guillemets (ou tirets), points de suspension, points d'interrogation, points d'exclamation; autant d'indicateurs dont on fait usage pour signaler que le sujet-agent ne fait que citer et reproduire dans sa propre allocution les arguments de son adversaire, sans les assumer mais en s'en distanciant, son but ultime étant de les disqualifier. Les parenthèses sont également présentes, tantôt avec une fonction purement illustrative, tantôt pour insérer des commentaires "axiologisés".

19 Les documents examinés font un large emploi de ces marques graphiques, comme le montre le tableau récapitulatif ci-dessous :

TIPA. Travaux interdisciplinaires sur la parole et le langage, 33 | 2017 
Tableau 1. Marques graphiques du DA et du DP

\begin{tabular}{|c|c|}
\hline \multirow[b]{2}{*}{$\begin{array}{l}\text { Doubles } \\
\text { guillemets }\end{array}$} & $\begin{array}{l}4 \text { fois dans le DA: } \\
\text { ex. "sperando nell'oscillazione borsistica" [en espérant dans les fluctuations du } \\
\text { marché boursier] (p. 1) } \\
\text { ex. "loro non erano usi a fare finanza creativa" [ils n'étaient pas habitués à } \\
\text { faire de la finance créative] (p. } 2 \text { ) }\end{array}$ \\
\hline & $\begin{array}{l}5 \text { fois dans le DP: } \\
\text { ex. Il documento della minoranza testualmente recita: "Per ristrutturare la Fortezza sono } \\
\text { contratti mutui (ed altri in fase di concessione) per circa } € 2.058 .000,00 \text { " [Le document de } \\
\text { la minorité déclare textuellement: "Pour restructurer la 'Fortezza', on a } \\
\text { contracté des emprunts (et d'autres en phase de concession) pour environ } \\
2.058 .000,00 € \text { "] (p. 1) } \\
\text { NB. On n'a pas pris en compte les cas où les guillemets sont employés avec une } \\
\text { fonction explicative, pour désigner des institutions, pour reprendre des } \\
\text { dénominations ou des expressions étrangères (tel est le cas, par exemple, du } \\
\text { proverbe latin "repetita juvant" dans le DP, p. } 2)^{9} \text {. }\end{array}$ \\
\hline \multirow{2}{*}{$\begin{array}{ll}\text { Points } & \text { de } \\
\text { suspension } & \end{array}$} & $\begin{array}{l}2 \text { fois dans le DA : } \\
\text { - la première à la fin d'un énoncé centré, en lettres capitales et en gras, pour lui } \\
\text { donner une plus grande emphase : } \\
\text { ex. LA REALTÀ È BEN DIVERSA... [LA RÉALITÉ EST BIEN DIFFÉRENTE...] } \\
\text { - la deuxième, sans aucune connotation, mais tout simplement pour ne pas } \\
\text { répéter le passage entier qui rappelle l'engagement pris par l'administration : } \\
\text { ex. “da parte del gestore a vigilare sul corretto svolgimento del servizio delegato...” } \\
\text { [de la part du gérant à veiller sur le déroulement correct du service délégué...] (p. } \\
\text { 2) }\end{array}$ \\
\hline & $\begin{array}{l}2 \text { occurrences dans le DP : } \\
\text { - respectivement une fois après le subordonnant adversatif « anzi », qui précède } \\
\text { la forme d'intensité exprimée par le superlatif de l'adjectif, dans un dicton de la } \\
\text { sagesse populaire: } \\
\text { ex. "Le bugie hanno le gambe corte, anzi...cortissime" [Les mensonges ne mènent } \\
\text { pas loin,... pas loin du tout, même] (p. 2) } \\
\text { - et une autre fois, pour éviter la reprise intégrale du texte cité : } \\
\text { ex. "Bello il Rifugio Miramonti ristrutturato, per il quale sono stati contratti mutui..." } \\
\text { [Qu'il est beau, le Refuge Miramonti restauré, pour lequel on a contracté des } \\
\text { emprunts...] (p. 2) }\end{array}$ \\
\hline
\end{tabular}




\begin{tabular}{|c|c|}
\hline \multirow{2}{*}{$\begin{array}{l}\text { Points } \\
\text { d'interrogation }\end{array}$} & $\begin{array}{l}6 \text { dans le DA : } \\
\text { Pour jalonner les questions pressantes par lesquelles le sujet-agent harcèle } \\
\text { indirectement le sujet-patient et polémique avec ses choix de gestion: } \\
\text { ex. Questo impegno economico [...] che utilità ha portato e porterà alle casse comunali } \\
\text { dato che per la struttura NON è stato previsto un piano di valorizzazione turistico } \\
\text { adeguato se non quello di darla in gestione praticamente gratis? [Cet engagement } \\
\text { économique [...] quelle utilité a-t-il apportée et apportera-t-il aux caisses } \\
\text { municipales, étant donné que, pour la structure, on n'a pas prévu de plan de } \\
\text { valorisation touristique adéquat si ce n'est celui de la donner en gérance à titre } \\
\text { gratuit ?] (p. 1) } \\
\text { ex. Forse sarà l'unico Comune italiano a non averci rimesso? [Peut-être que c'est la } \\
\text { seule mairie italienne qui n'a rien perdu ?] (p. 2) }\end{array}$ \\
\hline & $\begin{array}{l}1 \text { dans le DP : } \\
\text { ex. Forse questo recupero sta sullo stomaco a qualcuno che ha investito nel settore del } \\
\text { turismo e della ristorazione e che sperava di acquistare l'immobile? [Peut-être ce } \\
\text { recouvrement est-il resté sur l'estomac de quelqu'un qui a investi dans le secteur } \\
\text { du tourisme et de la restauration et qui espérait acheter l'immeuble ?] (p. 2) } \\
\text { Plus } 3 \text { points d'interrogation consécutifs entre parenthèses, référés ironiquement } \\
\text { à la préposition erronée della (au lieu de con la) dans un énoncé rapporté entre } \\
\text { guillemets: } \\
\text { ex. il documento contestato presenta una affermazione quale “è ovvia l'importanza di tale } \\
\text { struttura e siamo completamente d'accordo della (???) sua realizzazione" [le document } \\
\text { contesté présente l'affirmation que "l'importance de cette structure est évidente } \\
\text { et nous sommes tout à fait d'accord de (?? ?) sa réalisation"] (p. 2) }\end{array}$ \\
\hline \multirow[b]{2}{*}{$\begin{array}{l}\text { Points } \\
\text { d'exclamation }\end{array}$} & $\begin{array}{l}3 \text { points d'exclamation consécutifs dans le DA: } \\
\text { ex. (il ns. gruppo due anni fa aveva proposto di effettuare un test nell'abitato di } \\
\text { Caprignana, ma l'Amministrazione ci tacciò di fantasiosi!!!) [il y a deux ans, notre } \\
\text { groupe avait proposé d'effectuer un test dans l'agglomération de Caprignana, } \\
\text { mais l'administration nous taxa de fantaisistes!!!] (p. 2) }\end{array}$ \\
\hline & $\begin{array}{l}2 \text { points d'exclamation consécutifs dans le DP : } \\
\text { ex. Le entrate dichiarate corrispondono al vero e sono facilmente riscontrabili dalle } \\
\text { fatture emesse facendo una semplice operazione matematica che si chiama somma (1+1 = } \\
2 \text { sia ben inteso!!) [Les recettes déclarées correspondent à la vérité et on peut } \\
\text { facilement les vérifier à partir des factures émises au moyen d'une simple } \\
\text { opération mathématique qui s'appelle addition ( } 1+1=2 \text { bien entendu ! !)] (p. 3) } \\
\text { Ce signe graphique étant utilisé comme forme emphatique et ironique pour } \\
\text { souligner des concepts exprimés entre parenthèses. }\end{array}$ \\
\hline
\end{tabular}




\begin{tabular}{|c|c|}
\hline \multirow[b]{2}{*}{ Parenthèses } & $\begin{array}{l}6 \text { fois sur } 10 \text { dans le DA : } \\
\text { Pour renfermer des commentaires marqués axiologiquement, presque toujours } \\
\text { négatifs ou ironiques: } \\
\text { ex. per danni provocati dalle piogge (a loro dire straordinarie) [pour des dégâts } \\
\text { provoqués par les pluies (d'après eux, extraordinaires)] } \\
\text { ex. (vanto dell'Amministrazione Comunale) [(orgueil de l'administration municipale)] } \\
\text { (p. } 1 \text { ) } \\
\text { Exception faite pour une seule référence connotée positivement: } \\
\text { ex. (vanto della popolazione) [(orgueil de la population)] (p. 2) } \\
\text { Les } 4 \text { autres cas ayant une fonction illustrative. }\end{array}$ \\
\hline & 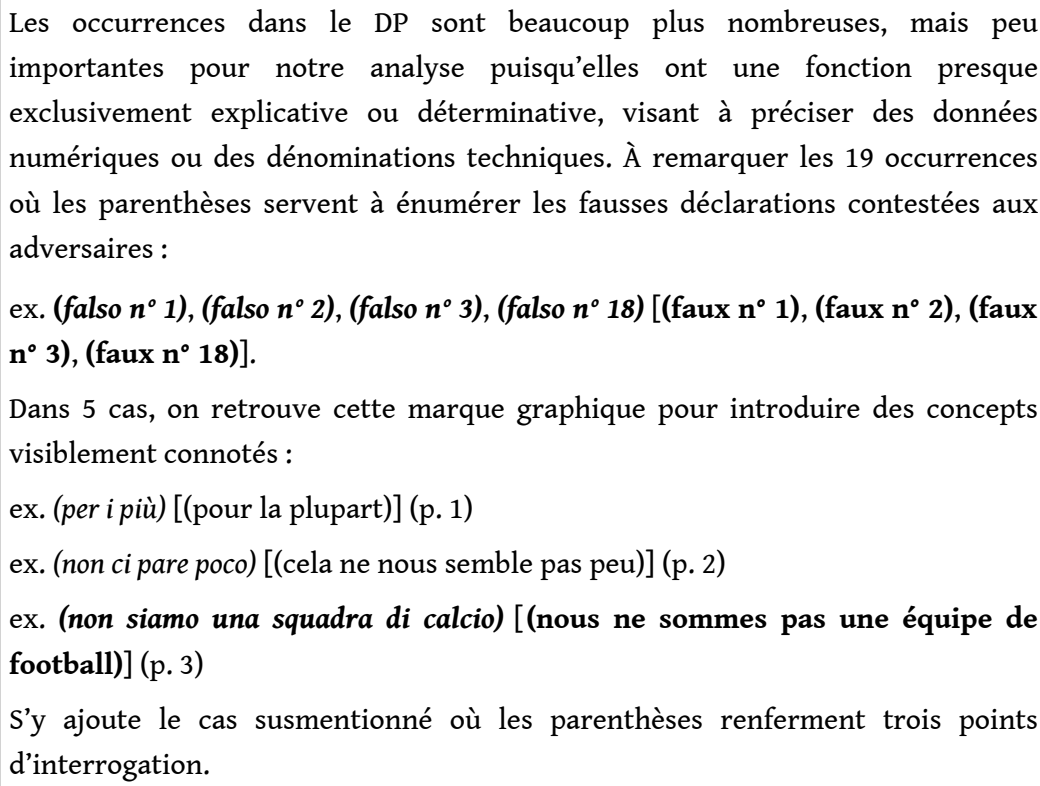 \\
\hline
\end{tabular}

Pour ce qui est du lexique, le discours conflictuel repose habituellement sur un réseau copieux de mots péjoratifs, voire injurieux, couvrant un registre fort varié ; en tant que reflets de l'activité conceptuelle des locuteurs, les choix lexicaux sont inévitablement révélateurs de leur prédisposition mentale.

21 Les documents en question sont émaillés d'expressions ayant une visée disqualifiante claire de la face des adversaires, sans pour autant tomber dans des jugements excessifs ou dans un vocabulaire outrageux. Cela témoigne de la volonté des acteurs sociaux, qui se trouvent dans une situation de polarisation, de soutenir leurs points de vue avec fermeté et rigueur, tout en gardant une conduite appropriée au contexte et au rôle officiel qu'ils jouent. D'ailleurs, la spécificité d'un échange polémique est qu'il ne s'agit pas d'un échange vif entre interlocuteurs animés par des sentiments violents, mais d'une confrontation de positions radicalement opposées (Amossy \& Burger, $2011: 12)^{10}$; un parti soutenu aussi par d'autres analystes: Le dénominateur commun des énoncés polémiques en tous genres n'est pas la violence mais le conflictuel (Garand, 1998 : 222).

Dans le DA, on peut signaler des expressions comme : 
una pessima programmazione finanziaria [une très mauvaise programmation financière] (p. 1) ; un "gioco finanziario" che eufemisticamente definiremo azzardato [un "jeu financier" que nous qualifierons, par euphémisme, de hasardeux] (p. 1) ; una qualità pessima dell'acqua [une très mauvaise qualité de l'eau] (p. 2)

des actes de langage informatifs qui exercent un rôle de contraste, elles sont parfois accompagnées de correctifs (emploi du "mais» de rectification) et de verbes de jugements, qui permettent au locuteur de contester les affirmations adverses :

La Corte dei Conti non ha rilevato irregolarità contabili gravi tali da richiedere controdeduzioni o giustificazioni del nostro operato [La Cour des comptes n'a pas relevé d'irrégularités comptables graves au point d'exiger des contre-déductions ou des justifications de nos actes] (p. 1).

Quindi termina questa gestione ma non è vero che l'Ostello chiuderà $i$ battenti in maniera definitiva (forse anche qui qualcuno in questi anni ha goduto delle varie difficoltà che ha incontrato questa struttura) [Ainsi, cette gérance se termine, mais il n'est pas vrai que l'auberge fermera ses portes de façon définitive (peut-être qu'ici aussi, quelqu'un s'est réjoui, ces dernières années, des diverses difficultés que la structure a rencontrées)] (p. 2).

L'impianto di teleriscaldamento non è in passivo (falso $\left.n^{\circ} 10\right)$ [L'installation du
télé-chauffage n'est pas déficitaire $\left(\right.$ faux $\left.\left.n^{\circ} 10\right)\right]($ p. 3).

Ciò, anche, al fine di attualizzare il principio fondamentale che il comune non $\grave{e}$

l'organo di pochi ma la casa di tutti [Cela, aussi, dans le but de mettre en œuvre le

TIPA. Travaux interdisciplinaires sur la parole et le langage, 33 | 2017 
principe fondamental que la mairie n'est pas l'organe de quelques-uns mais la maison de tous] (p. 4).

L'abbattimento del capitale sociale di SEVERA deliberato dalla assemblea dei soci comporta una perdita nel patrimonio e non nelle casse comunali (falso $n^{\circ} 17$ ) [L'abattement du capital social de SEVERA, délibéré par l'assemblée des actionnaires, entraîne une perte dans le patrimoine et non dans les caisses municipales (faux $\left.n^{\circ} 17\right)$ ] (p. 4).

Ce jeu de balançoire entre DA et DP montre clairement que :

L'objectif du discours des partisans et des adversaires est le même: s'imposer, vaincre, non seulement discursivement mais socialement et politiquement. [...] cette fin conditionne de manière déterminante la nature des discours; elle est pour quelque chose dans leur forme essentiellement polémique. (Windisch, $1982: 108$ )

\subsection{Plan discursif : stratégies en œuvre}

En ce qui concerne les stratégies discursives mobilisées dans l'acte verbal conflictuel, les procédés les plus récurrents identifiés par Windisch (1982) sont :

- la citation : on emploie tant le discours direct ou la citation proprement dite, consistant à reprendre explicitement le dire de l'adversaire, que le discours rapporté indirect, reposant sur des paraphrases introduites par des verbes déclaratifs ;

- la concession: on commence par valider la thèse de l'autre en faisant montre d'un acquiescement apparent à l'égard de ses arguments, pour rejeter ensuite l'opinion adverse avec plus de virulence ;

- la réfutation : on conteste les arguments de la thèse opposée en démontrant qu'ils ne sont pas pertinents ou qu'ils sont insuffisants ${ }^{11}$;

- l'ironie : on cherche à détruire les propos rivaux en les ridiculisant, avec une visée parodique et dévalorisante ;

- la simulation: on use à bon escient du contexte ou de la situation extralinguistique dans laquelle se déroule le conflit pour tourner en dérision l'adversaire ;

- le démasquage : on dévoile les points faibles ou les non-dits de la thèse de l'interlocuteur pour le déplacer dans une position fâcheuse aux yeux du public. Le démasquage fait contrepoids au masquage, qui représente l'autre face du discours conflictuel: on dissimule ou l'on occulte certains défauts, certains aspects idéologiques, ou des traits jugés négatifs de la personnalité du locuteur, qui tâche de séduire aux dépens de son concurrent ;

- la représentation fantasmatique : on reformule, tout en l'inversant, le discours de l'autre pour le disqualifier et le dénigrer;

- la stratégie de la guerre invisible: on n'énonce pas explicitement la cible de l'attaque verbale, mais on repère le caractère conflictuel du discours à travers les éléments situationnels extralinguistiques.

Au regard des deux discours examinés, l'analyse des marques graphiques nous a permis de relever que la citation est largement utilisée dans la joute verbale, aussi bien par le sujet-agent que par le sujet-patient qui, interpellé, passe à la contre-attaque et revêt le rôle d'agent. Le procédé discursif le plus fréquent dans le DA reste néanmoins le démasquage, visant à dévoiler tous les mauvais choix effectués par la majorité, en contraste avec les bonnes pratiques qu'une administration avisée est censée adopter. On enregistre également la présence de la concession, où le sujet-agent reconnaît 
d'abord la validité de l'initiative promue par l'administration (sujet-patient) pour en relever ensuite les failles, ainsi que de l'ironie, par laquelle on fait apparaître les adversaires comme incapables et ridicules.

Voici quelques exemples, suivis d'une représentation graphique des données :

Tableau 2. Stratégies discursives du DA

\begin{tabular}{|c|c|}
\hline Citati & $\begin{array}{l}\text { ex. Non aveva detto il capogruppo di Maggioranza, a mezzo stampa, che "loro non erano usi } \\
\text { a fare finanza creativa"? [Le chef de groupe de la majorité n'avait-il pas dit qu'“ils } \\
\text { n'étaient pas habitués à faire de la finance créative" ?] (p. 1) }\end{array}$ \\
\hline & 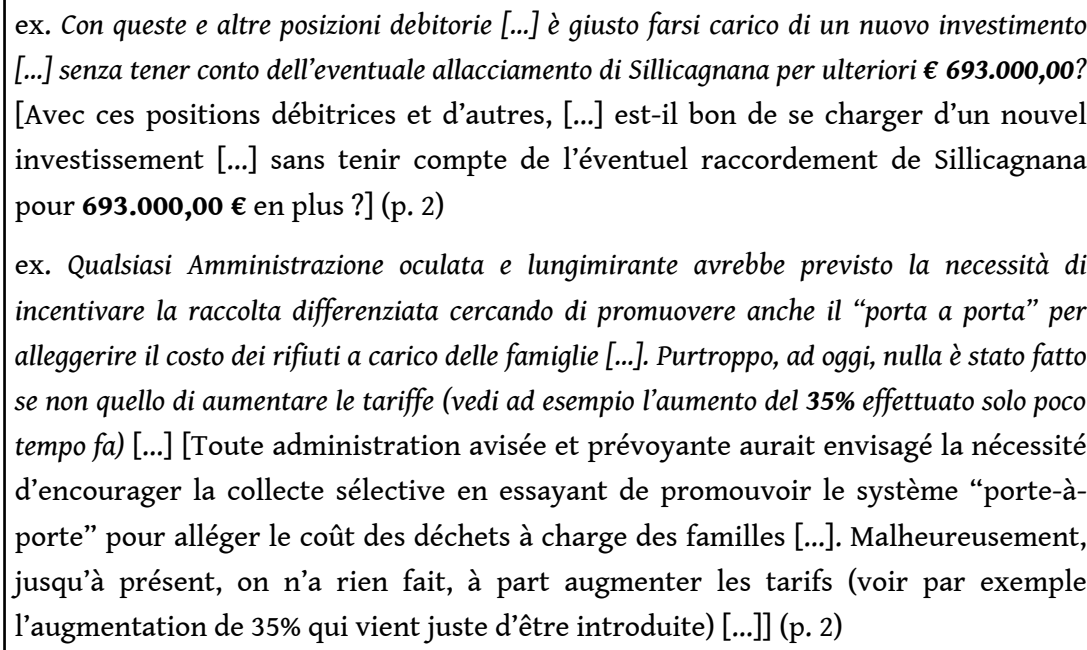 \\
\hline ssion & $\begin{array}{l}\text { ex. - Asilo Nido. È ovvia l'importanza di tale struttura e siamo completamente d'accordo della } \\
\text { sua realizzazione. Ma non si sono resi conto al momento della programmazione finanziaria } \\
\text { che quando finivano i contributi della Regione Toscana per GESTIRE la struttura ci si } \\
\text { indebitava di oltre } € 100.000,00 \text { annui? [- Crèche. L'importance de cette structure est } \\
\text { évidente et nous sommes tout à fait d'accord avec sa réalisation. Mais ne se sont-ils } \\
\text { pas rendu compte, lors de la programmation financière, qu'une fois les contributions } \\
\text { de la Région Toscane terminées, pour GÉRER la structure, on s'endettait pour plus de } \\
100.000,00 € \text { par an ?] (p. 1) }\end{array}$ \\
\hline Ironie (1) & $\begin{array}{l}\text { ex. (il ns. gruppo due anni fa aveva proposto di effettuare un test nell'abitato di Caprignana, } \\
\text { ma l'Amministrazione ci tacciò di fantasiosi!!!) [(il y a deux ans, notre groupe avait } \\
\text { proposé d'effectuer un test dans l'agglomération de Caprignana, mais } \\
\text { l'administration nous taxa de fantaisistes!!!)](p.2) }\end{array}$ \\
\hline
\end{tabular}


Figure 1. Pourcentage des stratégies du DA

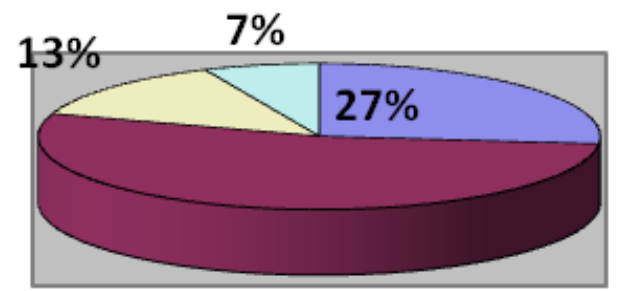

$53 \%$ $\square$ Citation

$\square$ Démasquage

$\square$ Concession

口Ironie
Concernant le discours du sujet-patient > agent, celui-ci annonce, dès le début, sa volonté de contester le document de l'adversaire pour faire connaître la vérité aux citoyens. En adoptant le mode argumentatif (on argumente sur la base de la valeur de vérité des arguments adverses), il reprend et dément l'une après l'autre, par des démonstrations circonstanciées et bien menées, les affirmations jugées infondées de la partie adverse ; associée à la citation, la réfutation s'annonce ainsi être la stratégie la plus fréquente, ce qui prouve que les énoncés polémiques, focalisés sur la dénégation du discours adverse, sont de par leur nature réfutatifs. Comme on l'a déjà signalé à propos du lexique, la fausseté est l'imputation principale à la charge des auteurs du document polémique, à qui on reproche aussi un manque d'intelligence face à des faits objectifs et pragmatiquement compréhensibles. La réfutation n'est jamais axiomatique, mais ponctuellement soutenue par des contre-arguments solides et des preuves sûres.

D'autres procédés sont également employés. Prête à répliquer aux accusations portées par la minorité (FTAs), la majorité a recours à la simulation pour montrer le côté bas de son adversaire politique. On cède parfois à la concession, mais ce n'est que pour démasquer et contester les assertions vicieuses du groupe adversaire; au moyen de l'ironie, on tend à accentuer le clivage entre l'intelligence du locuteur et la stupidité des antagonistes.

Au-delà des stratégies envisagées par Windisch (1982), une des modalités utilisées par le sujet-patient pour corroborer ses affirmations et légitimer ses actions, contre les accusations de la partie adverse, consiste à faire appel au sens commun et au bagage de connaissances de base que possède tout citoyen, de manière à souligner ultérieurement l'évidence des faits (on peut noter la prétérition dans le premier exemple de cette modalité). Et si cela ne suffit pas, les résultats sur le terrain sont là pour prouver la justesse des décisions prises :

Questa opera è risultata $2^{\circ}$ al Premio BioEnergy 2011 Best Practices, [...] scusate ma anche queste sono soddisfazioni di cui siamo fieri e che devono rendere orgogliosa la cittadinanza. [Cette réalisation a obtenu le deuxième Prix BioEnergy 2011 Best Practices, [...] excusez-nous mais ce sont là des satisfactions dont nous sommes fiers et dont les citoyens eux aussi doivent s'enorgueillir.] (p. 3)

Ci-dessous quelques exemples pour chaque procédé, suivis du graphique illustratif : 
Tableau 3. Stratégies discursives du DP

\begin{tabular}{|c|c|}
\hline Citation (5) & $\begin{array}{l}\text { ex. Ripetiamo quanto detto dal nostro capogruppo che "noi" non siamo abituati a giocare } \\
\text { con i soldi degli altri tanto meno con quelli dei nostri concittadini [Répétons ce qu'a dit } \\
\text { notre chef de groupe, que "nous" ne sommes pas habitués à jouer avec l'argent } \\
\text { d'autrui, encore moins avec celui de nos concitoyens] (p. 3) } \\
\text { Formules d'introduction récurrentes dans le DP : } \\
\text { ex. Il documento testualmente recita: “...” [Le document déclare textuellement: “...”] } \\
\text { Elles sont aptes à reprendre les données numériques ou les arguments fournis par } \\
\text { les adversaires pour prouver les irrégularités comptables présumées de } \\
\text { l'administration. }\end{array}$ \\
\hline & $\begin{array}{l}\text { ex. La falsità di tale affermazione ci sorprende e ci rimane incomprensibile, tanto da farci } \\
\text { dubitare dell'intelligenza di chi l'ha scritta, ove si tenga conto che tali soggetti hanno libero } \\
\text { accesso ai documenti comunali. [La fausseté de cette affirmation nous étonne et } \\
\text { reste pour nous incompréhensible, au point de nous faire douter de l'intelligence de } \\
\text { ceux qui l'ont écrite, si l'on tient compte du fait que ces sujets ont libre accès aux } \\
\text { documents municipaux] (p. 1) } \\
\text { ex. Ma anche in questo caso la bugia è grande come il Monte Tambura. Sia l'acquisto che la } \\
\text { sua ristrutturazione sono stati realizzati totalmente con contributi a fondo perduto per } \\
\text { un importo totale di } € \text { 789.000,00. [Mais dans ce cas aussi, le mensonge est gros } \\
\text { comme le Mont Tambura. Tant l'achat que sa restructuration ont été entièrement } \\
\text { réalisés avec des aides non remboursables, pour un montant de 789.000,00 } € \text { ] (p. } \\
\text { 2) } \\
\text { ex. Tale affermazione di per sé vera ed incontestabile, per la minoranza ci pare che sia solo di } \\
\text { facciata. } \\
\text { Invero, se tale opera è di primaria ed essenziale importanza per il benessere della } \\
\text { collettività, anche un eventuale esposizione debitoria è assolutamente necessaria e } \\
\text { comprensibile da chicchessia. [Il nous semble que cette affirmation, en elle-même vraie } \\
\text { et incontestable, n'est qu'une façade pour la minorité. } \\
\text { En fait, si l'on admet que cette structure est d'une importance première et } \\
\text { essentielle pour le bien-être de la collectivité, un éventuel endettement est } \\
\text { également tout à fait nécessaire et compréhensible par qui que ce soit] (p. 2) }\end{array}$ \\
\hline & $\begin{array}{l}\text { ex. Inoltre leggere il detto documento ci ha fatto piacere, in quanto ci offre l'opportunità di } \\
\text { far conoscere alla cittadinanza di quale bassissimo spessore sia l'opposizione nel nostro } \\
\text { Comune, che nella circostanza ha fatto un colossale autogol, come sarà provato con } \\
\text { documenti e fatti alla mano. I dati riportati sono totalmente privi di verità, cioè sono } \\
\text { falsi [...] [En outre, lire ce document nous a fait plaisir, car cela nous offre la } \\
\text { possibilité de faire savoir aux citoyens à quel point l'opposition de notre mairie est } \\
\text { misérable, elle qui, à cette occasion, a marqué un autogoal colossal, comme cela sera } \\
\text { démontré, documents et faits à l'appui. Les données rapportées sont complètement } \\
\text { dépourvues de vérité, autrement dit, fausses [...]] (p.1) }\end{array}$ \\
\hline
\end{tabular}




\begin{tabular}{|c|c|}
\hline & 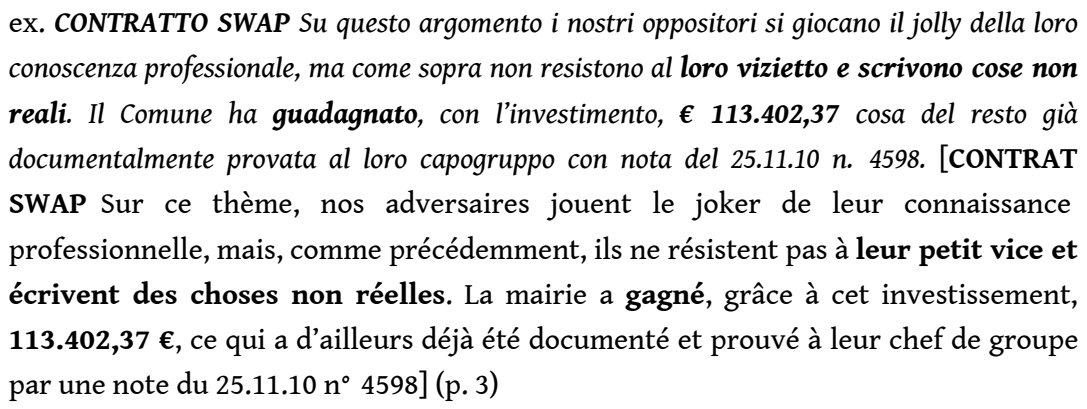 \\
\hline Ironie (2) & $\begin{array}{l}\text { ex. Le entrate dichiarate corrispondono al vero e sono facilmente riscontrabili }[\ldots] \\
\text { facendo una semplice operazione matematica che si chiama somma }(1+1=2 \text { sia ben } \\
\text { inteso!!) [Les recettes déclarées correspondent à la vérité et on peut facilement } \\
\text { les vérifier }[\ldots] \text { émises au moyen d'une simple opération mathématique qui s'appelle } \\
\text { addition }(1+1=2 \text { bien entendu !!)] }(\text { p. } 3)\end{array}$ \\
\hline $\begin{array}{l}\text { Appel au } \\
\text { bagage } \\
\text { cognitif des } \\
\text { citoyens (3) }\end{array}$ & $\begin{array}{l}\text { ex. Ognuno conosce la differenza tra un mutuo e un contributo a fondo perduto e non ci } \\
\text { pare sia il caso di dilungarci oltre. [Chacun connaît la différence entre un emprunt et } \\
\text { une aide non remboursable et il ne nous semble pas qu'il vaille la peine de } \\
\text { s'étendre davantage là-dessus.] (p. 1) } \\
\text { ex. Per ognuno è facile comprendere immediatamente il vantaggio per il Comune, che ha } \\
\text { saputo rendere attivo un immobile senza gravare sui cittadini e, nel contempo, ricavare } \\
\text { dallo stesso una entrata da utilizzare per la collettività. [Chacun peut comprendre } \\
\text { immédiatement l'avantage pour la mairie, qui a su rendre actif un immeuble sans } \\
\text { peser sur les citoyens et, en même temps, tirer de celui-ci une recette à utiliser } \\
\text { pour la collectivité] (p. 2) } \\
\text { ex. Ognuno sa che i mutui sono cosiddetti debiti che servono per finanziare opere da } \\
\text { realizzare per l'interesse della collettività. Ora in assenza di alchimie finanziarie a cui non } \\
\text { siamo abituati e di sponsors da cui farci finanziare [...], l'attività del Comune, che ha come } \\
\text { preciso obbligo quello di rispondere alle esigenze della collettività [...], richiede la necessità di } \\
\text { reperire risorse anche presso istituti finanziari per l'investimento. [Chacun sait que les } \\
\text { emprunts sont des dettes qui servent à financer des ouvrages qui doivent être } \\
\text { réalisés dans l'intérêt de la collectivité. Donc, faute d'alchimies financières } \\
\text { auxquelles nous ne sommes pas habitués et de sponsors qui puissent nous financer } \\
\text { [...], l'activité de la mairie, dont l'obligation ultime est de répondre aux exigences de } \\
\text { la collectivité [...], exige qu'on trouve des ressources, y compris auprès } \\
\text { d'établissements financiers pour l'investissement.] (p. 3) }\end{array}$ \\
\hline
\end{tabular}




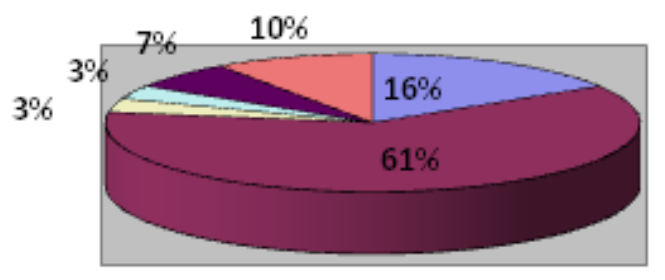

\begin{tabular}{|l|}
\hline$\square$ Citation \\
$\square$ Réfutation \\
$\square$ Simulation \\
$\square$ Concession \\
$\square$ Ironie \\
$\square$ Appel au bagage cognitif
\end{tabular}

En clôture du document, s'adressant directement aux citoyens en tant que destinataires principaux du discours, le sujet-patient a d'abord recours à un acte réparateur (FFA ou Face Flattering Act) en s'excusant pour la surcharge de données fournies, ensuite, il confirme une fois de plus la validité et l'efficacité des mesures adoptées :

Ci dispiace avervi rubato un po' di tempo con tale elenco di numeri ma se avrete la pazienza di leggere con attenzione avrete modo di capire che quello che l'Amministrazione ha realizzato e sta realizzando non è frutto di cattiva gestione, anzi rappresenta un insieme di opere di cui siamo orgogliosi e che molti altri avrebbero voluto sul loro territorio. [Nous sommes désolés de vous avoir fait perdre un peu de temps avec cette liste de chiffres, mais si vous avez la patience de lire avec attention, vous pourrez comprendre que ce que l'administration a réalisé et est en train de réaliser ne relève pas d'une mauvaise direction, mais représente, au contraire, un ensemble d'ouvrages dont nous sommes fiers et que bien d'autres auraient voulu mettre sur pied sur leur territoire.] (p. 4)

Enfin, il lance une dernière pique vigoureuse aux adversaires, que le caractère gras contribue à emphatiser :

non siamo disponibili ad accettare allusioni ed ammiccamenti di nessun tipo, tanto meno se fatti su basi assolutamente false. [nous ne sommes disposés à accepter aucune sorte d'allusions ou de clins d'œil, encore moins s'ils sont faits sur des bases tout à fait fausses.] (p. 4)

Des exemples jusqu'ici mentionnés il ressort que la dynamique conflictuelle du DA et du DP s'appuie sur différentes formes verbales, servant la visée argumentative de l'échange polémique : 
Tableau 4. Types d'énoncés du DA et du DP

\begin{tabular}{|l|l|}
\hline $\begin{array}{l}\text { Énoncés avec une } \\
\text { valeur } \\
\text { performative }\end{array}$ & $\begin{array}{l}\text { Avrebbe previsto, già deliberato, quanto promesso (DA), accettare (DP) : marqueurs } \\
\text { des actes illocutoires (commissifs, déclaratifs), ils traduisent la volonté des } \\
\text { parties en jeu de jouer un rôle actif dans le débat et, plus en général, dans } \\
\text { l'arène de l'administration publique. }\end{array}$ \\
\hline Verbes factifs & $\begin{array}{l}\text { Realizzare, guadagnare, ristrutturare, finanziare,... (DA et DP) : renvoyant à des } \\
\text { actions faites ou non faites par les parties antagoniques, ils véhiculent l'idée } \\
\text { d'un faire dans le discours. }\end{array}$ \\
\hline Verbes assertifs & $\begin{array}{l}\text { Dire, sottolineare, ripetere, informare,... (DA et DP) : employés dans des phrases } \\
\text { déclaratives, ils régissent des affirmations adressées aux citoyens ou } \\
\text { permettent de reprendre les arguments adverses, contestés et démentis. }\end{array}$ \\
\hline $\begin{array}{l}\text { Expressions } \\
\text { jugement }\end{array}$ & $\begin{array}{l}\text { Essere, suivi d'un adjectif (DA et DP) : on porte un jugement de valeur sur la } \\
\text { conduite des opposants. }\end{array}$ \\
\hline
\end{tabular}

On pourrait dire, pour terminer, que le déroulement d'un conflit discursif ressemble à ce qui se passe sur un échiquier, où chaque coup du joueur, résultat d'un raisonnement calme et attentif et d'une bonne, voire parfaite, maîtrise des règles du jeu, vise à protéger la reine et à mettre en échec l'adversaire.

\section{Le contre-discours de fin d'année de Beppe Grillo}

Comme on l'a précédemment anticipé, le discours conflictuel, actualisé dans plusieurs modalités discursives, est par sa nature même un contre-discours, qui reprend un autre discours afin de le disqualifier (Windisch, 2007).

Partant de ce constat, nous examinons de plus près un contre-discours officiel à vocation persuasive, où la communication verbale (les choix lexicaux), allant de pair avec les signifiants non verbaux (les images), vise à la réussite de la narration discursive. Il s'agit, en l'occurrence, du contre-discours que le fondateur du Mouvement 5 étoiles (Movimento 5 Stelle ou M5S), Beppe Grillo, a tenu sur le web le 31 décembre 2015, en même temps que le discours traditionnel de fin d'année du président de la République Sergio Mattarella. En fait, c'est un cas particulier de contre-discours, qui ne reprend pas à proprement parler les mots de l'interlocuteur, vu que les deux discours ont eu lieu en même temps, mais l'opposition réside dans le fait même que Grillo parle aux Italiens à un moment précis de l'année où il est coutume - une sorte de rituel - que seul le président de la République tienne son discours.

Un premier élément de discordance entre les deux acteurs sociaux est perceptible sur le plan visuel. Pour l'occasion, le président Mattarella, rompant avec la tradition respectée par ses prédécesseurs, ne prononce pas son discours d'usage au Quirinale (siège du président de la République), mais est assis dans le salon de son appartement, ce qui ne nuit en rien à l'autorité de son discours : une autorité et une légitimité qui lui sont conférées socialement par son rôle institutionnel.

Le cadre choisi par Beppe Grillo est tout à fait différent : il parle en direct streaming sur son blog, au centre d'une estrade avec un fond noir, sans aucun décor; il porte un 
veston clair, un pantalon foncé et une cravate, il est debout devant un pupitre, avec deux projecteurs fixés sur lui. Le frame joue sur le contraste figure-fond ${ }^{12}$, où l'élément au premier plan - l'acteur social/agent - est encore plus emphatisé au niveau visuel et conceptuel ; l'effet est celui d'un hologramme du locuteur qui parle en direct et c'est là justement le sens des mots de Grillo.

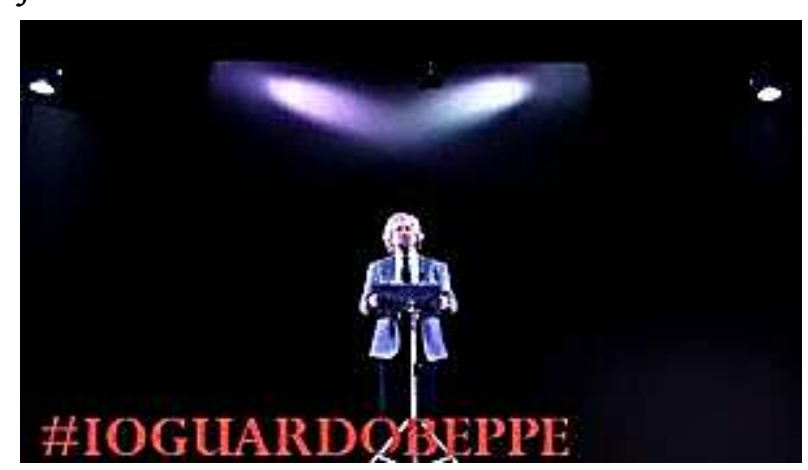

Discours de fin d'année 2015 de Beppe Grillo. ${ }^{13}$

Du point de vue strictement textuel, le discours du président Mattarella est focalisé sur les questions brûlantes de l'agenda du gouvernement, tant au niveau national - emploi, inégalités des chances (selon le genre ou les aires géographiques), pollution dans les grandes villes, spéculation et exploitation incontrôlée des ressources naturelles, laisser-aller et vandalisme qui pénalisent l'art et la culture, différentes formes de mafia - seulement à l'échelle internationale - terrorisme fondamentaliste en premier lieu, nécessité de garantir paix et stabilité, phénomènes migratoires. Les Italiens, affirme le Président, croient aux valeurs de la légalité et de l'honnêteté, et s'attendent à voir ces valeurs respectées, une tâche qui investit chaque citoyen :

Tutti siamo chiamati ad avere cura della Repubblica.

[Nous sommes tous appelés à prendre soin de la République.]

Sergio Mattarella exprime toute sa confiance dans le pays et dans son peuple, avec une attention particulière envers les femmes italiennes, chargées de multiples engagements et responsabilités. C'est un discours qui, tout en restant lucide et réaliste, se déroule sous le signe de l'espoir dans un avenir plus prometteur.

Il en va bien autrement du contre-discours de Grillo, qui dure environ trois minutes et qui est tout entier centré sur la dénonciation de la transformation du pays et de ses institutions en un hologramme ; Grillo inaugure ainsi la métaphore qui sera le fil rouge de son spectacle théâtral pendant sa tournée en 2016 :

Sono Beppe Grillo, anzi l'ologramma di Beppe Grillo, sono un fantasma.

[Je suis Beppe Grillo, ou mieux, l'hologramme de Beppe Grillo, je suis un fantôme.]

Il ne mentionne jamais le président du Conseil Matteo Renzi, ni le leader de Forza Italia Silvio Berlusconi, mais il s'en prend à la politique tout court qu'il juge fausse et artificielle. Il porte d'abord l'estocade contre le chef d'État Mattarella, bien que ses mots soient beaucoup moins violents comparés aux attaques directes contre son prédécesseur (il suffit de penser à son discours de fin 2014, quand il avait dit : Via Renzi e Napolitano! [Allez-vous-en, Renzi et Napolitano !], alors que cette fois, il se contente de parler en termes plus généraux) : 
Io non esisto. Come non esiste questo Paese, come non esiste il Parlamento, non esiste il governo. È tutto un ologramma. Abbiamo un Presidente della Repubblica che è l'ologramma di un ologramma (unico caso al mondo).

[Moi, je n'existe pas. De même que ce pays n'existe pas, de même que le Parlement n'existe pas, que le gouvernement n'existe pas. Tout est un hologramme. Nous avons un président de la République qui est l'hologramme d'un hologramme (seul cas au monde).]

Le terme ologramma, dépourvu de toute connotation morale positive, mais axiologiquement orienté vers la péjoration, est omniprésent tout au long du discours, avec 16 occurrences au total, oscillant entre singulier et pluriel; par la mise en valeur d'un concept-clé porté à l'attention du public, l'anaphore - une figure d'insistance typique du style oratoire - produit un effet percutant, aussi bien sur le plan sémantique que sur le plan sonore, renforçant le potentiel argumentatif du discours.

Après l'attaque contre le gouvernement et le président de la République, Grillo prend pour cible les députés et les sénateurs qu'il qualifie de proiezioni di qualcosa di morboso [projections de quelque chose de morbide], et puis, les référendums et les lois populaires, qui sont, eux aussi, des hologrammes. C'est plus précisément à deux parlementaires de Forza Italia, Maurizio Gasparri et Renato Brunetta, et au secrétaire fédéral de la Ligue du Nord, Matteo Salvini, de jouer le rôle de patients :

Vi sembra possibile che esista uno come Gasparri che è la proiezione del cavallo di Caligola?

Vi sembra normale che Salvini sia un leader e Brunetta un esponente del Parlamento italiano? Sono ologrammi venuti male.

[Vous semble-t-il possible qu'il existe un homme comme Gasparri, qui est la projection du cheval de Caligula ? Vous semble-t-il normal que Salvini soit un leader et Brunetta un représentant du Parlement italien ? Ce sont des hologrammes mal réussis.]

L'on voit comment, par la technique de la raillerie, visant à tourner en ridicule l'autre (Windisch, 2007 : 52), le locuteur dévalue ses adversaires, en contestant allègrement leur image et leur identité politique en vue de leur "décrédibilisation" (il s'agit bel et bien d'une réfutation de ces figures en tant que protagonistes de la scène politique italienne). Loin de ménager la face d'autrui, on cherche évidemment à la disqualifier et à la détruire de façon permanente (Charaudeau, 2013).

L'Italie même, toujours selon Grillo, est un hologramme :

Noi siamo un'espressione olografica, non esistiamo.

[Nous sommes une expression holographique, nous n'existons pas.]

Il en ressort un décalage flagrant entre le réel et l'illusoire, le dire et le faire des antagonistes :

Tutto è finto, tutto prendi e non ti rimane niente, 80 euro, referendum, niente rimane. [...] Non esiste più niente in questa politica incredibile. 
[Tout est faux, on prend tout et il ne reste rien, 80 euros, référendums, il ne reste rien. Il n'existe plus rien dans cette politique incroyable.] ${ }^{14}$

Tout en restant sous-entendue, la référence à Matteo Renzi, qui est le promoteur de ces initiatives (80 euros, référendums), est claire; l'usage de l'implicite évite la confrontation ouverte avec l'opposant politique. On a ici un type particulier de FTA, plus subtil et plus insinuant, qui ne se limite pas à porter atteinte à la face des sujets passifs (les adversaires politiques), mais qui va jusqu'à nier leur identité, leur existence même, comme le souligne la répétition dans le discours du verbe statif esistere, avec 8 occurrences, dont 7 à la forme négative.

Vient ensuite une âpre critique des médias, dont la remise en cause fait appel à un registre sarcastique :

I media vengono usati come ologramma di se stessi, viene usato il giornale per rendere vero un ologramma di chi ci scrive. Le televisioni, i mezzi di informazione che hanno rovinato $e$ continuano a rovinare questo Paese, è tutto un ologramma.

[Les médias sont utilisés comme un hologramme d'eux-mêmes, on utilise le journal pour rendre vrai l'hologramme de ceux qui y écrivent. Les télévisions, les moyens d'information qui ont ruiné et qui continuent à ruiner ce pays, tout est hologramme.]

Créant quelque chose à partir du rien, les médias sont coupables de donner une identité à ceux qui en sont démunis ; cela revient à ôter aux moyens de communication toute crédibilité. Dans cette liste noire, les banques ne pouvaient pas manquer, avec les créanciers, les produits dérivés, etc. : rien qu'un hologramme, comme tout le reste.

Le concept d'identité, qui est latent et sous-tend tout le discours, se configure finalement comme le grand absent du jeu social :

Noi stiamo diventando degli schizofrenici di noi stessi, copie di copie di copie di copie. [Nous sommes en train de devenir des schizophrènes de nous-mêmes, des copies de copies de copies de copies.]

Faute d'un original bien identifié, il n'y a qu'une série infinie de doubles.

On continue par une référence à la politique étrangère, notamment à ce qui s'est passé en Libye avec Kadhafi ; une des erreurs que le sujet-agent impute à la nation est de changer trop facilement de coalition, aujourd'hui comme dans le passé :

Ci alleiamo e chi si allea con noi si allea con un ologramma. Abbiamo visto nella Prima, nella Seconda Guerra Mondiale, abbiamo visto con Gheddafi... com'è andato a finire.

[Nous nous allions et celui qui s'allie avec nous s'allie avec un hologramme. Nous avons vu dans la Première, dans la Seconde Guerre mondiale, nous avons vu avec Kadhafi... comment il a fini.]

Par une série d'énoncés constatifs, correspondant, en fait, à des actes de langage implicites, des actes d'assertion (Austin, 1962), Grillo accomplit un acte de démasquage en vue de rétablir ce qu'il juge être la vérité des faits. 

stratégie de discours dont l'intention globale (Reboul \& Moeschler, 1998 : 191) est de démonétiser les principales institutions italiennes. Il se propose par là de secouer la torpeur de ses auditeurs et de susciter en même temps leur indignation, pour qu'ils prennent les rênes de leur vie et qu'ils aient une part active dans la gestion du pays. On 
retrouve ainsi les deux traits essentiels de Grillo, à savoir la vigueur polémique et l'engagement politique.

\section{Discussion}

66 À vouloir tracer un bref bilan au terme de notre analyse, nous remarquons avant tout que les stratégies et les procédés discursifs déployés au fil d'un discours conflictuel sont variés et représentent la clé de la réussite du combat verbal.

Les deux cas de figure examinés relèvent du même domaine, à savoir la sphère institutionnelle-politique, mais le premier s'inscrit d'emblée dans la typologie du discours dialogal, qui voit deux parties engagées dans un échange polémique jouant sur la polarisation sujet-agent/sujet-patient, alors que le deuxième est un contre-discours, ou bien, un discours polémique monogéré, qui vise à attaquer une cible sans que celleci rétorque (Amossy, 2011). Ils se distinguent également sur l'axe diamésique, puisqu'ils utilisent respectivement le canal écrit et oral.

Par rapport à l'échange des deux documents polémiques, parsemés de termes péjoratifs à forte charge affective (bassissimo spessore, atto scellerato, falso/falsità,...), le contrediscours de Grillo est caractérisé par l'absence du registre émotif au profit d'un lexique plus sec et plus dépouillé, mais immédiat et incisif, construit essentiellement autour du mot pivot ologramma et du syntagme verbal non esiste, des expressions ayant une connotation visiblement négative, répétées à intervalles réguliers de façon à provoquer un leitmotiv sémantique et sonore. Les verbes performatifs sont ici absents, ce qui démontre la visée surtout descriptive du message prononcé par le leader du M5S, qui dénonce les défauts des institutions italiennes par des actes de langage représentatifs et confie la force perlocutoire de son discours aux armes de l'ironie et de la satire, tout en sollicitant son auditoire par des formes d'interrogation. Dans l'échange polémique, les parties adverses ont recours à une variété plus ample d'actes de langage (assertifs, commissifs, déclaratifs), qui soutiennent la confrontation des thèses et la visée énonciative des interactants, en renforçant la potentialité argumentative de leurs propositions.

69 Du fait même qu'il s'agit de textes écrits plus longs et plus articulés que le contrediscours de Grillo, les documents de Garfagnana présentent un éventail plus large de stratégies discursives, telles que la citation, la réfutation, la simulation, le démasquage, l'ironie et la concession, autant de mécanismes qui sont tour à tour exploités par le sujet-agent et le sujet-patient pour mener l'offensive et la contre-attaque.

En définitive, il nous semble évident que la dynamique conflictuelle, notamment dans le cas du discours polémique, se dévoile à travers plusieurs facettes et formes discursives, dont le dénominateur commun est de déprécier l'adversaire, en ridiculisant sa face et en minant sa crédibilité (FTAs). La disqualification passe par des arguments appropriés et des axiologiques négatifs qui ne sont pas forcément violents ou vitupérants, en raison du statut des participants et du cadre communicationnel où se situe le discours; en effet, comme le soulignent Amossy (2008) et Maingueneau (2008), la violence verbale, tout en demeurant une marque importante de la polémique, ne constitue pas une composante indispensable de sa structure. 


\section{Pour conclure}

71 Si la communication est un enjeu scientifique et politique majeur du XXI e siècle (Wolton, 2001), en tant que condition essentielle de l'intercompréhension et d'une coopération mutuelle basée sur le respect d'autrui, cela est d'autant plus vrai que les rapports sociaux et institutionnels reposent souvent sur des actes de langage conflictuels qui entravent la négociation communicative. C'est là, d'ailleurs, le propre de la démocratie, dont la nature conflictuelle constitue un trait indéniable, d'après la philosophe belge Chantal Mouffe, inspiratrice du mouvement Podemos. Celle-ci [la démocratie] ne doit pas chercher le consensus, mais permettre l'expression de positions antagonistes, a-t-elle déclaré lors d'une interview à L'Obs, en avril 2015.

72 Comme cela a été illustré par les quelques exemples présentés, qui nous ont permis de mieux appréhender la morphologie du discours conflictuel, il s'agit d'un type de discours [...] comportant des règles et des contraintes spécifiques (Windisch, 1987 : 57). Les différentes modalités discursives sont modulées et déclinées en fonction du contexte situationnel où interagissent les acteurs sociaux, qui alternent dans le rôle d'agent et de patient. De la sorte, une réaction en chaîne est enclenchée, dans laquelle les antagonistes s'affrontent avec les mots et s'attaquent réciproquement, en s'efforçant coup sur coup de remporter la victoire, autrement dit, de triompher sur l'autre, de le dénigrer aux yeux du public par des actes de langage dévalorisants et d'imposer enfin leur propre thèse, manipulant les récepteurs et conquérant leur adhésion.

De plus, ainsi que nous l'avons observé à propos du deuxième cas de figure analysé, il y a un autre aspect dont il faut tenir compte, à savoir la mise en scène, l'impact communicatif du plan visuel (contexte et langage corporel) qui, allant de pair avec le plan verbal, concourt à véhiculer le message voulu et s'intègre parfaitement à la construction stratégique du discours.

74 Le pouvoir des mots n'étant que trop évident, on comprend bien que l'enjeu consiste finalement surtout dans l'usage qu'on en fait et dans les stratégies discursives qu'on met en œuvre. Le succès d'un discours tient indubitablement à la capacité de tirer profit des différents outils langagiers et au choix des procédés argumentatifs les plus appropriés, encore plus lorsqu'il s'agit d'une interaction conflictuelle, caractérisée, de par sa nature, par une dualité dichotomique entre deux parties, dont chacune cherche à l'emporter sur l'autre.

\section{BIBLIOGRAPHIE}

Amossy, R. (2008) Modalités argumentatives et registres discursifs : le cas du polémique, in Gaudin-Bordes, L. \& G. Salvan (eds) in Les registres. Enjeux pragmatiques et visées stylistiques, Louvain-la-Neuve: Academia-Bruylant, p. 93-108. 
Amossy, R. (2011) La coexistence dans le dissensus. La polémique dans les forums de discussion, Semen [en ligne], 31, Polémiques médiatiques et journalistiques, p. 25-42. Disponible sur : http:// semen.revues.org/9051 (consulté le 12 juin 2017).

Amossy, R. \& M. Burger (2011) Introduction : la polémique médiatisée, Semen [en ligne], 31, Polémiques médiatiques et journalistiques, p. 7-24. Disponible sur : http://semen.revues.org/ 9072 (consulté le 13 juin 2017)

Austin, J.L. (1962) How to do things with words, Oxford: Oxford University Press Austin, J.L. (1970) Quand dire, c'est faire, traduit de l'anglais par G. Lane, Paris: Éditions du Seuil. Bart, C. (1998) Le discours politique, Paris: Presses Universitaires de France.

Breton, P. (2000) La parole manipulée, Paris: La Découverte Poche.

Brown, P. \& P. Levinson (1987) Politeness, Cambridge: Cambridge University Press.

Charaudeau, P. (2002) A quoi sert d'analyse le discours politique?, Análisi del discurs politic [en ligne], Barcelone: IULA-UPF. Disponible sur : http://www.patrick-charaudeau.com/A-quoi-sertd-analyse-le-discours.html (consulté le 12 juin 2017).

Charaudeau, P. (2013) L'arme cinglante de l'ironie et de la raillerie dans le débat présidentiel de 2012, Langage et société, 146, p. 35-47.

Dascal, M. (1998) Types of polemics and types of polemical moves, in Cmejrkova, S. et al. (eds) Dialogue Analysis VI, Proceedings of the 6th Conference, 1, 1996, Prague. Tübingen: Max Niemeyer, p. 15-33.

Felman, S. (1979) Le discours polémique (Propositions préliminaires pour une théorie de la polémique), Cahiers de l'Association internationale des études françaises [en ligne], 31, p. 179-192. Disponible sur : http://www.persee.fr/doc/caief_0571-5865_1979_num_31_1_1195 (consulté le 12 juin 2017).

Garand, D. (1998) Propositions méthodologiques pour l'étude du polémique, in Hayward, A. \& D. Garand (eds) États du polémique, Montréal: Nota Bene, p. 211-268.

Goffman, E. (1967) Interaction rituals: Essays on face to face behaviour, New York: Anchor Books. Grice, H.P. (1975) Logic and Conversation, in Cole, P. \& J.L. Morgan (eds) Syntax and semantics. Speech acts, vol. III, New York-London: Academic Press, p. 41-58.

Grice, H.P. (1979) Logique et conversation, traduit de l'américain par F. Berthet et M. Bozon, Communications, 30 , p. 57-72.

Kerbrat-Orecchioni, C. (1980) La polémique et ses définitions, in Gelas, N. \& C. Kerbrat-Orecchioni (eds) Le discours polémique, Lyon: Presses Universitaires de Lyon, p. 3-40.

Kerbrat-Orecchioni, C. (1986) « Nouvelle communication » et « analyse conversationnelle », Langue française, 70, p. 7-25.

Kerbrat-Orecchioni, C. (2008) Les actes de langage dans le discours. Théorie et fonctionnement, Paris: Armand Colin.

Lagorgette, D. (2012) Insulte, injure et diffamation : de la linguistique au code pénal ?, Argumentation et analyse du discours [en ligne], 8, p. 1-14. Disponible sur : https://aad.revues.org/ 1312 (consulté le 12 juin 2017).

Lakoff, G. \& M. Johnson (1980) Metaphors we live by, traduit de l'américain par M. de Fornel en collaboration avec J.-J. Lecercle, Chicago: The University of Chicago. 
Langacker, R.W. (1999) Assessing the cognitive linguistic enterprise, in Janssen, T. \& G. Redeker (eds) Cognitive linguistics: Foundations, scope and methodology, Berlin/New York: Mouton de Gruyter, p. 13-59.

Maingueneau, D. (1983) Sémantique de la polémique. Discours religieux et ruptures idéologiques au XVII ${ }^{e}$ siècle, Lausanne: L'Âge d'Homme.

Maingueneau, D. (2008) Les trois dimensions du polémique, in Gaudin-Bordes, L. \& G. Salvan (eds) Les registres. Enjeux pragmatiques et visées stylistiques, Louvain-la-Neuve: Academia-Bruylant, p. 109-120.

Maingueneau, D. (2007, 2010 pour la présente impression) Analyser les textes de communication, deuxième édition entièrement revue et augmentée (1re éd. 1998), Paris: Armand Colin.

Moeschler, J. (1982) Dire et contredire. Pragmatique de la négation et acte de réfutation dans la conversation, Berne: Peter Lang.

Moïse, C. \& A. Oprea (2015) Présentation. Politesse et violence verbale détournée, Semen [en ligne], 40, Politesse et violence verbale détournée. Disponible sur : https://semen.revues.org/ 10387 (consulté le 12 juin 2017).

Oléron, P. (1995) Sur l'argumentation polémique, Hermès [en ligne], 16, Argumentation et rhétorique II, p. 15-27. Disponible sur : http://documents.irevues.inist.fr/bitstream/handle/ 2042/15179/HERMES_1995_16_15.pdf?sequence=1 (consulté le 12 juin 2017).

Plantin, C. (2002) Des polémistes aux polémiqueurs [en ligne], in Declercq, G. et al. (eds) La parole polémique, Paris: Honoré Champion, p. 377-408. Disponible sur : www.icar.cnrs.fr/pageperso/ cplantin/documents/2002e.doc (consulté le 12 juin 2017).

Reboul, A. \& J. Moeschler (1998) La pragmatique aujourd'hui. Une nouvelle science de la communication, Paris: Éditions du Seuil.

Santone, L. (2014) Quand la politique tire la langue : le Vaffa Day du M5S (Movimento 5 Stelle), Mots. Les langages du politique, 106, p. 87-103.

Searle, J.R. (1972) Les actes de langage. Essai de philosophie du langage, traduit de l'anglais par H. Pauchard, Paris: Hermann.

Windisch, U. (1982) Pensée sociale, langage en usage et logiques autres. L'exemple de la causalité dans la vie quotidienne en acte, Lausanne: L'Âge d'Homme.

Windisch, U. (2007) Le K.-O. verbal. La communication conflictuelle, nouvelle édition (1 ${ }^{\text {re }}$ éd. 1987), Lausanne: L'Âge d'Homme.

Wolton, D. (2001) La communication, un enjeu scientifique et politique majeur du XXI ${ }^{\mathrm{e}}$ siècle, L'année sociologique [en ligne], 51, 2, Presses Universitaires de France, p. 309-326. Disponible sur : https://www.cairn.info/revue-l-annee-sociologique-2001-2-page-309.htm (consulté le 12 juin 2017).

Wolton, D. (2009) Informer n'est pas communiquer, Paris: CNRS (Débats).

\section{Sitographie}

Discorso di fine anno 2015 di Beppe Grillo [en ligne]. Disponible sur : http://www.beppegrillo.it/ 2015/12/ discorso_di_fin_3.html (consulté le 9 mai 2016).

Grillo e il contro-discorso di Capodanno: "L'Italia non esiste" [en ligne]. Disponible sur : http:// www.repubblica.it/politica/2016/01/01/news/grillo_e_il_contro-

discorso_di_capodanno_l_italia_non_esiste_-130446080/ (consulté le 9 mai 2016). 
Messaggio di fine anno del Presidente della Repubblica Sergio Mattarella [en ligne]. Disponible sur : http://www.quirinale.it/elementi/Continua.aspx?tipo=Discorso\&key=215 (consulté le 12 mai 2016).

Nuit debout et le "refus du leader" : "Occuper une place ne suffit pas" [en ligne]. Disponible sur : http:// bibliobs.nouvelobs.com/idees/20160422.OBS9048/nuit-debout-et-le-refus-du-leader-occuperune-place-ne-suffit-pas.html (consulté le 21 mai 2016).

Scambio di documenti polemici fra maggioranza e minoranza [en ligne]. Disponible sur : http:// www.comune.san-romano-in-garfagnana.lu.it/?p=565 (consulté le 7 mai 2016).

\section{NOTES}

1. C'est là l'approche que nous adopterons dans cet article, sans pour autant vouloir prétendre que ce soit la seule position possible ; en effet, comme le remarque Plantin (2003 : 383), on peut évidemment argumenter, et se disputer, en l'absence de toute expérience guerrière, la guerre n'étant pas forcément le modèle de toute opposition.

2. Les archétypes des rôles (role archetypes) ou rôles sémantiques fondamentaux - agent, patient, instrument, expériencieur - reviennent en linguistique cognitive en tant qu'instruments cognitifs qui permettent d'analyser les processus mentaux et linguistiques des locuteurs (Langacker, 1999: 40 sqq.). En d'autres termes, selon Langacker, les notions grammaticales universelles - sujet, objet, nom, verbe et possessifs - sont des instanciations d'habiletés cognitives innées et sont caractérisées schématiquement par les archétypes conceptuels enracinés dans notre expérience d'interaction avec le monde : l'agent, soit l'élément proéminent, l'actant déclencheur d'un procès, est de préférence le sujet, alors que le patient, celui ou ce qui change d'état sous l'effet du procès, sert le plus souvent d'objet.

3. La théorie des faces (le mot face désignant métonymiquement la personne et métaphoriquement son identité) a été développée par Brown et Levinson (1987) qui, à leur tour, ont repris et systématisé les travaux du sociologue américain Goffman (1967). Chaque individu est censé posséder deux faces, en tant que composantes complémentaires mais parfois incompatibles, qui peuvent déclencher le conflit : une face positive (l'image sociale valorisante qu'on revendique pour soi) et une face négative (son propre territoire - corps, vêtements, vie privée - qu'il faut défendre des intrusions d'autrui). Kerbrat-Orecchioni (2008: 75) observe que l'apport majeur de cette théorie est d'envisager les actes de langage par rapport aux effets qu'ils peuvent avoir sur les faces des parties en présence.

4. On parle de manipulation cognitive (Breton, 2000: 101): on manipule le contenu cognitif du message lui-même.

5. Rappelons qu'une argumentation a deux fonctions dominantes : une fonction persuasive (thèse soutenue), lorsque l'émetteur cherche à convaincre le récepteur de la vérité de sa thèse et à capter son estime ou sa sympathie, et une fonction polémique (thèse rejetée), lorsque l'émetteur vise à détruire la thèse adverse, en en montrant les limites.

6. Windisch (2007 : 26-27) préfère les termes plus forts de discours / sujet manipulateur et de discours / sujet manipulé, plus aptes, à son avis, à qualifier les modifications et les déformations 
que l'auteur d'un discours conflictuel fait subir au discours adverse lors de la lutte discursive, jusqu'à parvenir au K.-O. verbal.

7. Il est à noter que l'auteur d'un discours conflictuel a deux interlocuteurs distincts : son adversaire et le public-témoin du conflit. La communication argumentative prévoit en effet trois rôles actanciels : le Proposant conteste le discours de l'Opposant devant un Tiers à convaincre (Plantin, 2003).

8. Les documents sur lesquels va se focaliser notre analyse sont accessibles par le biais de la sitographie finale.

9. De même, on n'a pas retenu les tirets en emploi énumératif et non comme marques du discours rapporté.

10. Là où Kerbrat-Orecchioni (1980) et Plantin (2003) mettent l'accent sur l'engagement émotionnel (passion, indignation, violence verbale) en tant que trait distinctif du discours polémique, Amossy et Burger (2011 : 11) jugent qu'on peut polémiquer sans recours aux passions. La polémique peut en effet s'exprimer avec retenue lorsque le statut des participants, les cadres communicationnels et les normes de politesse en vigueur dans la communauté ou le genre choisi l'exigent.

11. En reprenant la typologie élaborée par Moeschler (1982: 87 sqq.), Windisch (2007:47) distingue trois types de réfutation ou de négation : la rectification (on réajuste le propos asserté par l'adversaire, considéré par le sujet-agent comme faux ou trop faible), la réfutation propositionnelle (plutôt que de corriger une assertion, on vise à nier la validité du contenu de l'énoncé adverse, au moyen d'une justification ou d'une explication), la réfutation présuppositionnelle (on rejette les présupposés politiques ou idéologiques d'autrui).

12. Le principe perceptif du figure/ground, que la sémantique cognitive a emprunté à la psychologie de la Gestalt, envisage un élément perceptuellement proéminent (figure ou 'profil') qui est mis en relief et se distingue du reste (ground ou 'fond'). Ce système de perception et de conceptualisation prédispose à une interprétation donnée.

13. Blog de Beppe Grillo : http://www.beppegrillo.it/2015/12/discorso_di_fin_3.html.

14. Grillo fait allusion à certaines initiatives du gouvernement, en particulier aux référendums proposés aux citoyens et au bonus de 80 euros, destiné aux salariés ayant un revenu annuel inférieur ou égal à 26000 euros.

15. Du mot vulgaire vaffanculo, «va te faire foutre ». Pour un approfondissement sur le Vaffa-Day du M5S, voir Santone (2014).

16. Selon la tripartition de Windisch (2007: 95), on peut identifier: le discours affectifconflictuel, le discours didactique-conflictuel et le discours ironique-conflictuel.

\section{RÉSUMÉS}

À l'heure actuelle, de nombreux échanges langagiers se placent sous le signe du désaccord, de l'antagonisme, de l'agressivité verbale, témoignant du fait que le conflit est, de gré ou de force, une composante incontournable de la réalité sociale. Un tel constat est à l'origine de la métaphore conceptuelle, envisagée par Lakoff et Johnson (1980, tr. fr. 1985) et répandue dans la culture occidentale, selon laquelle l'activité discursive est structurée en termes de dispute, d'affrontement de thèses; une sorte de duel verbal au cours duquel le sujet-agent développe ses propres argumentations, en réfutant, voire en ridiculisant le discours de l'adversaire ou sujetpatient. Du fait qu'il reprend un autre discours pour s'y opposer, le discours conflictuel se profile comme un contre-discours, visant d'un côté à frapper la face de l'autre pour en proposer une 
représentation sociale péjorative et, de l'autre côté, à consolider l'image de crédibilité et de légitimité du locuteur. Dans cet article, nous nous proposons de réfléchir, en nous appuyant sur deux cas de figure en italien (un échange polémique et un contre-discours), sur les mécanismes langagiers enclenchés lors d'une interaction conflictuelle, en focalisant notamment notre attention sur les stratégies discursives que les acteurs sociaux mettent en œuvre pour gagner le combat verbal (Windisch, 1982, 2007).

In a contemporary context, many linguistic exchanges are marked by various forms of antagonism, of disagreement, of verbal aggressiveness, confirming that conflict is a component of social reality that cannot be ignored. This explains the conceptual metaphor, proposed by Lakoff and Johnson (1980, fr. tr. 1985) and widespread in Western culture, according to which speech activity is structured in terms of debate, clash, and theses; a sort of verbal duel during which social actors occupy in turn the position of Agent and Patient.

The conceptually prominent participant who speaks and wants to obtain the audience's support to his/her thesis (Agent) develops his argumentation by objecting to the discourse of his/her adversary (Patient); that is to say, he/she absorbs, manipulates, and deforms the opposing thesis so as to place it in an unfavorable light, or he opposes his/her adversary's argument using a counter-argument which disproves it. For his/her part, the subject who is involved in the dispute and suffers from the attack is pushed to react, to retort to his/her interlocutor, by transforming himself/herself into an Agent and adopting the same discourse strategies. This dynamics triggers a chain reaction with an alternation of Agent-discourse and Patient-discourse (Windisch, 1982; Maingueneau, 1983) in a discourse conflict whose aim is the legitimization of the former discourse and the delegitimization of the latter.

Since conflictual discourse takes up another discourse with the purpose of objecting to it, the former is also envisaged as a counter-discourse, aimed, on the one hand, at metaphorically slapping the other's face to give a depreciatory social representation of him/her (FTAs or Face Threatening Acts), and, on the other hand, at strengthening his/her own image of credibility and legitimacy.

Based on two Italian case studies relating to the political-institutional domain, the current paper reflects on linguistic mechanisms engaged during a conflictual interaction, especially focusing on textual features and discourse strategies which social participants make use of in order to win the verbal battle, according to the model elaborated by Windisch $(1982,2007)$.

The first case study consists in a polemical exchange, whose primary pragmatic aim is to discredit the contender involved in the dispute and his/her discourse (Kerbrat-Orecchioni, 1980). With regard to the textual form, among the main linguistic elements found in the two examined documents, we count the graphic signs of indirect speech: quotation marks (or dashes), suspension points, question marks, exclamation marks; signs used to point out that the Agentsubject limits him/herself to quoting and to reproducing in his/her own allocution his/her adversary's arguments, by no means accepting them but keeping his/her distance from them, his/her only purpose being to discredit his/her adversary. Brackets are used too, sometimes with a purely explanatory function, sometimes to introduce some axiological remarks.

As for lexicon, conflictual discourse is built on a rich repertoire of pejorative words which cover a varying register; reflecting the speaker's conceptual activity, lexical choices give evidence of his/ her mental attitude. In our specific case study, the two documents are sprinkled with derogatory expressions aiming to denigrating the antagonists, yet without producing extreme judgments or outrageous vocabulary, in accordance with the style of polemical exchanges whose main feature is not verbal violence but the confrontation of conflicting positions and opinions (Dascal, 1998; Garand, 1998; Amossy \& Burger, 2011).

As far as discourse strategies are concerned, the most prominent ones are: 
- quotation: speaker 1 (S1 - Agent-subject) uses direct speech or quotation in its strict sense, which consists in voicing explicitly the words of the adversary ( $\mathrm{S} 2$ - Patient-subject) as well as indirect speech, resting on paraphrases preceded by introducing verbs;

- concession: S1 validates S2's thesis showing an apparent compliance with his/her arguments, but solely in order to reject the opposing opinion with more virulence;

- refutation: S1 opposes the arguments of his/her adversary's thesis proving that they are not relevant or are inadequate;

- irony: S1 disparages his/her antagonist under the disguise of praise; indeed, he/she pretends to share his/her rival's purposes in order to better denigrate them, with a parodic and depreciating aim;

- simulation: S1 uses advisedly the context or the extra-linguistic situation in which conflict takes place so as to make his adversary ridiculous;

- unmasking: S1 reveals the weak points and the unspoken elements of his/her interlocutor's discourse to show him/her in a negative light. Unmasking counterbalances masking, which is the other side of a conflictual discourse: some faults, ideological aspects or negatively judged traits of S1's personality are dissimulated or hidden, in order to score points against the competitor.

Our second case study, belonging to the genre of ironical-conflictual discourse (Windisch, 2007), is represented by the counter-discourse of the Five Star Movement's founder Beppe Grillo, which was broadcast on his blog on 31 December 2015, when and while Italian President Sergio Mattarella was delivering his end-of-year message. As we show in our analysis, linguistic devices such as irony, parody and satirical register turn out to be very efficient means, capable of supporting argumentation, devaluing the antagonist and undermining his/her credibility. Another important aspect that has to be taken into account is the communicative impact of the visual level which, going together with the verbal one, contributes to transmit the planned message and integrates perfectly with strategic speech construction.

In conclusion, the power of words being evident, the stakes lie finally in their use and in the strategies brought into play. The success of a discourse depends upon the speaker's capacity to take advantage of a variety of linguistic tools and to choose the most appropriate discursive devices, still more when the interaction is conflictual, characterized essentially by a dichotomous dualism between two parts, each of which tries to prevail over the other.

\section{INDEX}

Mots-clés : communication conflictuelle, échange polémique, discours-agent et discourspatient, contre-discours, stratégies discursives

Keywords : conflictual communication, polemical exchange, counter-discourse, Agent and Patient discourses, discourse strategies

\section{AUTEUR}

\section{ALESSANDRA ROLLO}

Université du Salento (Lecce, Italie)

alessandra.rollo@unisalento.it 